\title{
Weak-Field Laser Phase Modulation Coherent Control of Asymptotic Photofragment Distributions
}

\author{
A. García-Vela \\ Instituto de Física Fundamental, Consejo Superior de Investigaciones Científicas, \\ Serrano 123, 28006 Madrid, Spain
}

\begin{abstract}
Coherent control of the asymptotic photofragment state-resolved distributions by means of laser phase modulation in the weak-field limit is demonstrated computationally for a polyatomic molecule. The control scheme proposed applies a pump laser field consisting of two pulses delayed in time. Phase modulation of the spectral bandwidth profile of the laser field is achieved by varying the time delay between the pulses. The underlying equations shows that such a phase modulation is effective in order to produce control effects on the asymptotic, long-time limit photofragment distributions only when the bandwidths of the two pulses overlap in a frequency range. The frequency overlap of the pulses gives rise to an interference term which is responsible for the modulation of the spectral profile shape. The magnitude of the range of spectral overlap between the pulses becomes an additional control parameter. The control scheme is illustrated computationally for the asymptotic photofragment state distributions produced from different scenarios of the $\mathrm{Ne}-\mathrm{Br}_{2}$ predissociation. Experimental application of the control scheme is found to be straightforward.
\end{abstract}

E-mail: garciavela@iff.csic.es 


\section{Introduction}

Exerting control of a molecular process to the extent of being able to steer its evolution towards a desired target has been a goal actively pursued in the last years. A variety of coherent control strategies has been developed in order to achieve such a goal, taking advantage of the coherent nature of light and of the quantum properties of the molecular systems involved. ${ }^{1,2}$ The control schemes designed have been applied to several molecular processes, using both weak and strong laser fields. ${ }^{3-25}$ While the different control targets pursued have been successfully achieved in general, several challenges still remain in the field of coherent control.

One of these challenges is to achieve effective control of the different observable properties of a molecular process by applying only phase modulation of the laser pulse in the weak field, one-photon regime. An advantage of weak laser fields is that they do not alter the inherent dynamics of the system. They also avoid undesired multiphoton ionization and fragmentation processes of the system that may occur with strong fields. Phase modulation of a fixed bandwidth laser pulse in the weak field regime is typically achieved by inducing a positive or negative chirp in the pulse. The positive (negative) chirp causes the central frequency of the pulse to increase (decrease) with time. As a result, while an unchirped pulse excites all the frequencies contained in its bandwidth simultaneously at every time, a positively (negatively) chirped pulse excites the lower (higher) frequencies of its bandwidth at earlier times and the higher (lower) frequencies at later times. The temporal delay in the excitation of the different frequencies of the pulse spectral width induced by the chirp can be used to modify the time evolution of a molecular process.

The issue of control of the state distributions of photodissociation fragments by means of phase modulation of a fixed bandwidth laser pulse in the weak field limit has been addressed in several works. ${ }^{26-33}$ A theoretical proof showed that for closed systems, phase control of the asymptotic, long-time limit final photofragment state distributions is not possible in the weak-field regime. ${ }^{26,27}$ Indeed, that proof showed that asymptotic product distributions are independent on the laser phase, 
and thus phase modulation cause no effects on the final fragment distributions. ${ }^{26,27}$ The reason, in brief, is that the laser phase distribution is a complex exponential function of the pulse freqeuencies, and since the fragment state distributions are square magnitudes, this complex function cancels out. An experiment on weakfield coherent control of retinal isomerization reported later on observed long-time control effects of the isomerization yields by modulating the laser phase. ${ }^{28}$ In order to clarify the apparent controversy between this experimental finding and the previous theoretical proof, the conditions for one-photon coherent phase control in isolated and open quantum systems were established. ${ }^{29}$ Based on these conditions, it was concluded that the modes involved in retinal isomerization can be considered as a subsystem coupled intramolecularly to the environment of the remaining modes of this large molecule, and under these "open" system conditions, weak-field coherent phase control of the subsystem properties is possible.

In addition, it has been shown that when a superposition of nonorthogonal, interfering states, like overlapping resonances, is prepared, transient weak-field phase control of the time-dependent populations of some electronic states in different systems is possible during a postpulse time window. ${ }^{30,31}$ Recently, it was reported the first computational demonstration of coherent control of the state distribution of photodissociation fragments based on pure phase modulation of the pump pulse in the weak-field regime. ${ }^{32,33}$ The specific process investigated was the vibrational

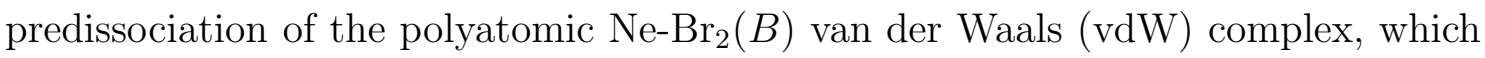
is excited to a superposition of overlapping resonance states using fixed bandwidth pulses with different linear chirps. Phase control effects on the transient vibrational populations of the $\mathrm{Br}_{2}\left(B, v_{f}\right)$ fragment produced upon predissociation were demonstrated by varying the chirp rate, and such control effects were found to persist for a long-time window of about 200 ps after the pulse is over. The postpulse longtime window of phase control effects on the $\operatorname{Br}_{2}\left(B, v_{f}\right)$ fragment populations was attributed to the change of the mechanism of interference between the $\mathrm{Ne}^{-\mathrm{Br}_{2}(B)}$ overlapping resonances when the chirp rate varies. ${ }^{32,33}$ 
Even in the cases of long-time windows of postpulse phase control effects on the transient fragment distributions, ${ }^{32,33}$ such effects eventually vanish at some point in time. Thus, the works reported so far demonstrate that, when the system is initially in a single eignestate, coherent control of asimptotic final state photofragment distributions by means of phase modulation of a single fixed bandwidth pulse in the weak-field regime is indeed not possible. In contrast, it has been recently reported that when the system is intially in a superposition state of (at least) two eigenstates, phase modulation of a single fixed bandwidth pulse used to excite the system in the weak-field regime can lead to control effects of the asimptotic photofragment distributions due to interference. ${ }^{34}$

However, the phase modulation achieved by inducing a chirp on a single Gaussianlike pulse is not the only way to modulate the phase of a laser field (from now on we shall assume a Gaussian-like shape for all the pulses for simplicity, although actually this assumption is not necessary). One can also modulate the phase distribution of the laser field by applying two Gaussian-like pulses with different carrier frequencies and a time delay between them. By varying the time delay between the two pulses one can mimic the effect of the single pulse chirp, because the frequencies of each of the two pulses are excited at different times. The bandwidth of the laser field composed of the two pulses remains fixed, since it is independent on the time delay between the pulses. It is noted that the possibility of modifying the spectral profile, and therefore the relative populations of the different states excited within a superposition, by varying the time delay between two subsequent pulses has been previously demonstrated experimentally ${ }^{10,35}$ and applied theoretically ${ }^{36}$ for atomic and diatomic systems using two identical pulses. In this work the above control scheme is applied for the first time to a realistic model of a polyatomic molecule, in order to control the asymptotic final state-resolved photofragment distributions, which are more complex than in diatomic molecules. Specifically, the control scheme

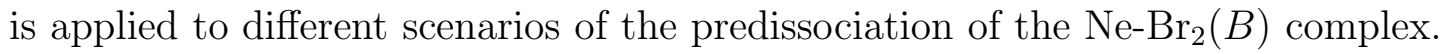

\section{Theoretical background}




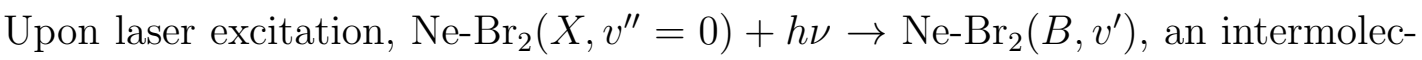
ular vdW resonance or a superposition of resonances of $\mathrm{Ne}_{-} \mathrm{Br}_{2}\left(B, v^{\prime}\right)$ is populated. Then the resonance or resonances excited decay to the fragmentation continuum through vibrational predissociation, $\mathrm{Ne}_{-\mathrm{Br}_{2}}\left(B, v^{\prime}\right) \rightarrow \mathrm{Ne}+\mathrm{Br}_{2}\left(B, v_{f}<v^{\prime}\right)$. This process has been studied in detail both experimentally ${ }^{37,38}$ and theoretically. ${ }^{39-42}$ The process of $\mathrm{Ne}_{-} \mathrm{Br}_{2}\left(B, v^{\prime}\right)$ excitation with a laser pulse and the subsequent predissociation of the complex was simulated with a full three-dimensional wave packet method (assuming $J=0$ ) described in detail elsewhere. ${ }^{40,43}$ It is noted that the lifetime calculated with the present theoretical model for the decay of the Ne$\mathrm{Br}_{2}\left(B, v^{\prime}=16\right)$ ground intermolecular resonance has been found to be $69 \mathrm{ps},{ }^{44}$ while the corresponding lifetime estimated experimentally is $68 \pm 3$ ps. ${ }^{38}$ This good agreement with the experimental lifetime implies that both the three-dimensional wave packet method and the potential surfaces used in the present simulations are quite realistic in order to describe this resonance decay process.

The pump laser field used in this work is a combination of two Gaussian pulses

$$
\mathcal{E}_{\text {pump }}(t)=\mathcal{E}_{0} e^{-\left(t-t_{1}\right)^{2} / 2 \sigma^{2}} \cos \left[\omega_{1}\left(t-t_{1}\right)+\phi_{1}\right]+\mathcal{E}_{0} e^{-\left(t-t_{2}\right)^{2} / 2 \sigma^{2}} \cos \left[\omega_{2}\left(t-t_{2}\right)+\phi_{2}\right],
$$

where $\omega_{1}, \omega_{2}$ and $t_{1}, t_{2}$ are the frequency and time centers, respectively, of the two pulses. In order to make simpler the interpretation of the results, the only source of phase modulation of the laser field of eqn (1) will be the time delay between the two pulses, $\Delta t=t_{2}-t_{1}$, and then the phases are taken to be $\phi_{1}=\phi_{2}=0$. Similarly for simplicity, the amplitudes of the pulses are assumed to be equal, $\mathcal{E}_{0}=1.0 \times 10^{-6}$ a.u., which corresponds to a maximum pulse intensity of about $3.5 \times 10^{4} \mathrm{~W} / \mathrm{cm}^{2}$, within the weak-field regime. A field like that of eqn (1) with two pulses separated by $\Delta t$ has been previously used ${ }^{45-47}$ to control the intensity of interference between two $\mathrm{Ne}_{-} \mathrm{Br}_{2}\left(B, v^{\prime}\right)$ overlapping resonances, with the aim of enhancing their lifetime. Such a field provides a large flexibility and degree of control for that purpose, by varying the time delay $\Delta t$, which can be readily achieved experimentally. 
We are now interested in the frequency amplitude profile $\tilde{\mathcal{E}}_{\text {pump }}(\omega)$,

$$
\tilde{\mathcal{E}}_{\text {pump }}(\omega)=\int_{-\infty}^{\infty} \mathcal{E}_{\text {pump }}(t) e^{i \omega t} d t
$$

because the photofragment state distributions depend on the square of $\tilde{\mathcal{E}}_{\text {pump }}(\omega)$, $\left|\tilde{\mathcal{E}}_{\text {pump }}(\omega)\right|^{2}$,

$$
P(E, n)=\mathcal{C}\left|\tilde{\mathcal{E}}_{\text {pump }}\left(\omega_{E}\right)\right|^{2} \lim _{t \rightarrow \infty}\left|\left\langle E, n\left|\exp \left(-i H_{B} t / \hbar\right)\right| \phi_{X}\right\rangle\right|^{2},
$$

where $E$ is the total energy of the fragments and the final fragment states are labeled by a global quantum number $n,\left|\phi_{X}\right\rangle$ is the initial eigenstate (e.g., the vibronic ground state) at energy $\epsilon_{1}$ times the projection of the transition dipole moment along the polarization direction of the field, $\omega_{E}=\left(E-\epsilon_{1}\right) / \hbar$ is the excitation frequency, $H_{B}$ is the nuclear Hamiltonian of the excited electronic state, and $\mathcal{C}$ is a constant. For a single Gaussian-like pulse $\mathcal{E}_{\text {pump }}(t)$ we have that $\tilde{\mathcal{E}}_{\text {pump }}(\omega)=A(\omega) e^{i \Phi(\omega)}$, and the phase dependence $e^{i \Phi(\omega)}$ vanishes in $\left|\tilde{\mathcal{E}}_{\text {pump }}(\omega)\right|^{2}=|A(\omega)|^{2}$, leading to an asymptotic $P(E, n)$ state distribution which is independent on the pulse phase dependence. Thus, no phase modulation control of the asymptotic, final $P(E, n)$ is possible in this case.

Now, for the field of eqn (1), we can perform analytically the integral of eqn (2), and after some algebra we find

$$
\begin{aligned}
\tilde{\mathcal{E}}_{\text {pump }}(\omega)= & \frac{\mathcal{E}_{0}\left(2 \pi \sigma^{2}\right)^{1 / 2}}{2}\left[\left(e^{-\sigma^{2}\left(\omega+\omega_{1}\right)^{2} / 2}+e^{-\sigma^{2}\left(\omega-\omega_{1}\right)^{2} / 2}\right) e^{i \omega t_{1}}+\right. \\
& \left.\left(e^{-\sigma^{2}\left(\omega+\omega_{2}\right)^{2} / 2}+e^{-\sigma^{2} i\left(\omega-\omega_{2}\right)^{2} / 2}\right) e^{i \omega t_{2}}\right]
\end{aligned}
$$

or in more compact form

$$
\tilde{\mathcal{E}}_{\text {pump }}(\omega)=A\left(\omega, \omega_{1}\right) e^{i \omega t_{1}}+B\left(\omega, \omega_{2}\right) e^{i \omega t_{2}} .
$$

Then

$$
\begin{gathered}
\left|\tilde{\mathcal{E}}_{\text {pump }}(\omega)\right|^{2}=A^{2}\left(\omega, \omega_{1}\right)+B^{2}\left(\omega, \omega_{2}\right)+A\left(\omega, \omega_{1}\right) B\left(\omega, \omega_{2}\right)\left[e^{i \omega\left(t_{2}-t_{1}\right)}+e^{-i \omega\left(t_{2}-t_{1}\right)}\right]= \\
A^{2}\left(\omega, \omega_{1}\right)+B^{2}\left(\omega, \omega_{2}\right)+2 A\left(\omega, \omega_{1}\right) B\left(\omega, \omega_{2}\right) \cos \left[\omega\left(t_{2}-t_{1}\right)\right] .
\end{gathered}
$$


The term $2 A\left(\omega, \omega_{1}\right) B\left(\omega, \omega_{2}\right) \cos \left[\omega\left(t_{2}-t_{1}\right)\right]$ of the power spectrum $\left|\tilde{\mathcal{E}}_{\text {pump }}(\omega)\right|^{2}$ of eqn (6) is a phase dependence that can be modulated just by varying the time delay $\Delta t=t_{2}-t_{1}$ between the two pulses of $\mathcal{E}_{\text {pump }}(t)$. Such a modulation will change the profile of $\left|\tilde{\mathcal{E}}_{\text {pump }}(\omega)\right|^{2}$, but still keeping the same fixed spectral bandwidth. A change in the $\left|\tilde{\mathcal{E}}_{\text {pump }}(\omega)\right|^{2}$ profile will involve in general a modification of the asymptotic $P(E, n)$ state distribution [see eqn (3)], thus making possible its coherent phase control.

The most important implication of eqn (6) is that the appearance of the phase dependence term is independent on the system to which the laser field is applied, which makes this control scheme of universal application. One of the main goals of the present work is to establish the conditions under which this type of control can be exerted on the asymptotic photofragment state-resolved distributions. In the following we shall analyze the different scenarios where this phase dependence term can lead to phase modulation coherent control of the asymptotic photofragment distributions in the general case of two different pulses in the field of eqn (1).

In the general case, $\omega_{1} \neq \omega_{2}$ in the field of eqn (1). However, it is stressed that a phase dependence is present in $\left|\tilde{\mathcal{E}}_{\text {pump }}(\omega)\right|^{2}$ also when $\omega_{1}=\omega_{2}$ (in this case $A\left(\omega, \omega_{1}\right)=B\left(\omega, \omega_{2}\right)$, and $\left|\tilde{\mathcal{E}}_{\text {pump }}(\omega)\right|^{2}=2 A^{2}\left(\omega, \omega_{1}\right)[1+\cos (\omega \Delta t)]$, as long as $\Delta t \neq 0$ (i.e, $\left.t_{1} \neq t_{2}\right)$. In the specific case of $t_{1}=t_{2}(\Delta t=0$ and $\cos (\omega \Delta t)=1)$ the phase dependence vanishes, and this situation would be equivalent to that of an unchirped single Gaussian-like pulse.

The phase dependence term $2 A\left(\omega, \omega_{1}\right) B\left(\omega, \omega_{2}\right) \cos (\omega \Delta t)$ is actually an interference term that arises when $\tilde{\mathcal{E}}_{\text {pump }}(\omega)$ is squared. Therefore, the requirement for this term to be nonzero is that the bandwidths $A\left(\omega, \omega_{1}\right)$ and $B\left(\omega, \omega_{2}\right)$ of the two pulses of the field of eqn (1) must overlap in a certain range of frequencies $\omega$, that coincides with the spectral range of the states populated in the superposition prepared. If there is no overlap between $A\left(\omega, \omega_{1}\right)$ and $B\left(\omega, \omega_{2}\right)$ (i.e., $\left.A\left(\omega, \omega_{1}\right) B\left(\omega, \omega_{2}\right)=0, \forall \omega\right)$, we have the situation of a sum of two independent, nonverlapping pulses, and the phase dependence in $\left|\tilde{\mathcal{E}}_{\text {pump }}(\omega)\right|^{2}$ vanishes same as in the single Gaussian-like pulse 
case. However, if there is overlap between the spectral bandwidths of the two pulses, the $\left|\tilde{\mathcal{E}}_{\text {pump }}(\omega)\right|^{2}$ intensity profile can be modulated in the range of frequency overlap by varying the time delay $\Delta t$ between the pulses. The frequency overlap requirement is always fulfilled in the case that $\omega_{1}=\omega_{2}$ (regardless that both pulses have the same bandwidth or not). In the general case that $\omega_{1} \neq \omega_{2}$, the spectral overlap condition is fulfilled only when the two bandwidths are large enough in comparison with the frequency difference $\Delta \omega=\omega_{2}-\omega_{1}$. It is noted that, as long as the pulses overlap in a frequency range, the position of the central frequencies and therefore their separation $\Delta \omega=\omega_{2}-\omega_{1}$ can be varied, becoming an additional control parameter. It should be pointed out here that a control scheme that uses two pulses delayed in time, namely a pump and a probe pulse with different carrier frequencies has been applied to control the dissociative ionization of $\mathrm{H}_{2} \cdot{ }^{19,48,49}$ By varying the time delay between the pump and probe pulses, the dissociation dynamics changes from adiabatic to nonadiabatic, with the corresponding variation of the fragment outcome. While this control method is related to the present one to some extent, its spirit is actually different.

To summarize the above discussion, the requirement to achieve coherent phase control of the asymptotic photofragment state distributions is the overlap of the two pulses of the pump laser field in the range of frequencies associated with the different states populated in the superposition created. By fulfilling this condition the $\left|\tilde{\mathcal{E}}_{\text {pump }}(\omega)\right|^{2}$ profile can be modulated in that frequency range by simply varying the time delay between the pulses, which is routinely done in current pump-probe experiments. In the following this will be illustrated by applying a laser field like that of eqn (1), to prepare a superposition of resonance states in $\mathrm{Ne}^{-} \mathrm{Br}_{2}\left(B, v^{\prime}\right)$, when the two pulses have both nonoverlapping and overlapping spectral bandwidths. The $\mathrm{Ne}-\mathrm{Br}_{2}\left(B, v^{\prime}\right)$ complex is used here because it is a system rich in different types of resonance states, which makes it appropriate for the present test purposes. In this sense, the effect on the phase control of the fact that the resonances populated in the superposition overlp between them or not, is also investigated. 


\section{Results and discussion}

When a superposition of resonance states of $\mathrm{Ne}^{-\mathrm{Br}_{2}}\left(B, v^{\prime}\right)$ is prepared, there are two possible situations, namely that the resonances overlap between them or not. Overlapping resonances are nonorthogonal states that can interfere between themselves, while nonoverlapping resonances cannot interfere. It was found previously ${ }^{32,33}$ that such overlapping resonance interference leads to significant long-time postpulse control effects on the transient photofragment populations, which are not found in the case of superpositions of nonoverlapping resonances. Thus, the two situations of overlapping and nonoverlapping resonances will be investigated here. The $\mathrm{Ne}-\mathrm{Br}_{2}\left(B, v^{\prime}=27\right)$ and $\mathrm{Ne}-\mathrm{Br}_{2}\left(B, v^{\prime}=16\right)$ systems will be used to prepare a superposition of overlapping and nonoverlapping resonances, respectively.

It has been shown that the ground intermolecular resonance of $\mathrm{Ne}^{-} \mathrm{Br}_{2}\left(B, v^{\prime}=\right.$ 27) overlaps with some vdW orbiting resonances corresponding to the lower $v^{\prime}-1$ vibrational manifold of $\mathrm{Br}_{2}(B) .{ }^{41,42}$ These orbiting resonances lie above the $\mathrm{Ne}+$ $\mathrm{Br}_{2}\left(B, v^{\prime}-1=26, j^{\prime}=0\right)$ dissociation threshold, and are supported by centrifugal barriers. In particular, the $v^{\prime}$ ground resonance overlaps mainly with a $v^{\prime}-1$ orbiting resonance located $\sim 1.2 \mathrm{~cm}^{-1}$ above in energy. The excitation spectrum of the Ne$\mathrm{Br}_{2}\left(B, v^{\prime}=27\right)$ ground resonance ${ }^{32}$ displays a main peak located at $-61.80 \mathrm{~cm}^{-1}$ (relative to the $\operatorname{Br}_{2}\left(B, v^{\prime}=27, j^{\prime}=0\right)+$ Ne dissociation threshold), associated with the $v^{\prime}$ ground vdW resonance, and several other overlapping peaks associated with $v^{\prime}-1$ orbiting resonances. The second most intense feature in the spectrum, located at $-60.63 \mathrm{~cm}^{-1}$, corresponds to the $v^{\prime}-1$ orbiting resonance separated from the $v^{\prime}$ ground resonance by $1.2 \mathrm{~cm}^{-1}$. Thus, a superposition of these two overlapping resonances located at -61.80 and $-60.63 \mathrm{~cm}^{-1}$ is prepared by tunning the pulse frequencies $\omega_{1}$ and $\omega_{2}$ of the field $\mathcal{E}_{\text {pump }}(t)$ to excite each of the resonances, respectively, from the $\mathrm{Ne}-\mathrm{Br}_{2}\left(X, v^{\prime \prime}=0\right)$ ground vibronic state.

\section{Spectrally nonoverlapping pulses}


We will examine first the scenario where the spectral bandwidths of the two pulses of $\mathcal{E}_{\text {pump }}(t)$ do not overlap. To this purpose, a field $\mathcal{E}_{\text {pump }}(t)$ with two pulses with a temporal full width at half-maximum (FWHM) of $\tau_{F W H M}=\sigma \sqrt{8 \ln 2}=100$ ps for each pulse, has been used. For these pulses the spectral FWHM is about $0.2 \mathrm{~cm}^{-1}$. For simplicity, in the simulations $t_{1}$ is always fixed at $t_{1}=0$ and the center $t_{2}$ of the other pulse is the only one varied, being $\Delta t=t_{2}$. In Fig. 1(a) the temporal profiles of the two pulses are shown at $t_{1}=0$ and at different values of $t_{2}$. In Fig. 1(b) the spectral bandwidth $\left|\tilde{\mathcal{E}}_{\text {pump }}(\omega)\right|^{2}$ (calculated numerically as the squared Fourier transform of $\mathcal{E}_{\text {pump }}(t)$, using eqn (2)) is displayed, showing that indeed the bandwidths of the two pulses do not overlap.

By applying the above laser field the vibrational predissociation of the $\mathrm{Ne}-\mathrm{Br}_{2}(B)$ system prepared in the superposition of the two $v^{\prime}=27$ and $v^{\prime}-1$ overlapping resonances is simulated. The normalized vibrational populations of the $\operatorname{Br}_{2}\left(B, v_{f}\right)$ product fragment are calculated as

$$
P_{v_{f}}^{n o r m}(t)=\frac{P_{v_{f}}(t)}{\sum_{v_{f}} P_{v_{f}}(t)},
$$

with $v_{f}=v^{\prime}-1, v^{\prime}-2, \ldots$. The $P_{v_{f}}(t)$ populations include the contributions of all the resonance states populated in the superposition prepared, and are equivalent to the transient populations measured in a time-resolved experiment. The transient vibrational populations for $v_{f}=v^{\prime}-1$ at times $t \geq 0$ are shown in Fig. 2(a) for different time delays $\Delta t=t_{2}$ between the two pulses of $\mathcal{E}_{\text {pump }}(t)$. As expected, the transient populations change to a large extent in time, depending on the time delay between the pulses. However, in all cases the populations converge to the same asymptotic final population of nearly 0.1. Thus, although there are effects of interference between the resonances present in the transient populations as a result of phase modulation caused by varying $\Delta t$, the asymptotic population cannot be controlled by phase modulation using the above field, as predicted by the theory.

The magnitude of the interference effects on the transient populations can be estimated in the following way. If the two $v^{\prime}$ and $v^{\prime}-1$ resonances are excited independently (i.e., each of them alone, in the absence of the other one), at $t_{1}=0$ 
and $t_{2}=\Delta t$, respectivley, they cannot interfere with each other. By summing the transient populations associated with these two independent simulations we get $P_{v_{f}}^{\text {norm,ind }}(t)=P_{v_{f}}^{n o r m,\left(v^{\prime}\right)}(t)+P_{v_{f}}^{n o r m,\left(v^{\prime}-1\right)}(t)$. Thus, in the population $P_{v_{f}}^{\text {norm,ind }}(t)$ the effect of interference between the resonances has been removed. Now the magnitude $\left(P_{v_{f}}^{n o r m}(t)-P_{v_{f}}^{n o r m, i n d}(t)\right) / P_{v_{f}}^{n o r m, i n d}(t)$ provides the effect of interference (in percentage) on the transient populations. This magnitude is displayed in Fig. 2(b) for the different time delays in the case of $v_{f}=v^{\prime}-1$.

All the curves show oscillations around zero that decrease in intensity and finally vanish at long times. Such oscillations are the signature of the effect of interference in the transient populations. The intensity of interference gradually vanishes as the two resonances decay in time. ${ }^{33}$ For each time delay there is typically a time window of postpulse (after the two pulses are over) effects of about 200 ps. This result is very similar to the previous finding using a single pump pulse to prepare the resonance superposition. ${ }^{32}$ The most intense postpulse effects $(\sim 0.65 \%)$ in Fig. 2(b) occur for $\Delta t=0$, which is the case where interference between the resonances is maximized because they are populated simultaneously during the longest time window. As the time delay between the pulses increases, the maximum intensisty of the oscillations decreases as a result of a decreasing intensity of interference. Nevertheless, the intersity of interference in the present case is remarkably smaller than in the previous work, ${ }^{32}$ because here the narrower spectral bandwidth [see Fig. 1(b)] excites a narrower range of overlapping energy components of the resonances. But the most interesting finding of Fig. 2 is that phase modulation of both the present laser field with two pulses nonoverlapping in the frequency domain and of the previous single pulse ${ }^{32}$ lead to the same qualitative result, namely that phase control effects are only possible for the transient populations in a long potpulse time window, but not for the asymptotic values of the distributions.

\section{Spectrally overlapping pulses}

Let us now consider the other scenario where the two pulses of the laser field 
of eqn (1) do overlap in the frequency domain. This scenario is found when the temporal width of the pulses is decreased with respect to the case of Fig. 1(a). In the present application a width of $\tau_{F W H M}=10$ ps has been used for both pulses. Similarly as in Fig. 1(a), in Fig. 3(a) the temporal profiles of the two pulses are shown for different time delays. In Fig. 3(b) the spectral profile $\left|\tilde{\mathcal{E}}_{\text {pump }}(\omega)\right|^{2}$ (again calculated numerically using eqn (2)) is displayed for several time delays between the two pulses of $\mathcal{E}_{\text {pump }}(t)$.

In all cases the bandwidth of $\left|\tilde{\mathcal{E}}_{\text {pump }}(\omega)\right|^{2}$ remains fixed, but the shape of the profile changes remarkably by varying $\Delta t$. For $\Delta t \neq 0$ the spectral profile display oscillations which are due to the term $2 A\left(\omega, \omega_{1}\right) B\left(\omega, \omega_{2}\right) \cos (\omega \Delta t)$ of eqn $(6)$, and more specifically to the $\cos (\omega \Delta t)$ factor of that term. The separation between the maxima of the oscillations is proportional to $1 / \Delta t$. Thus, for small $\Delta t$ the profile presents very few oscillations (e.g., for $\Delta t=10$ and 20 ps we see only two and four oscillations in the corresponding profiles, respectively), while the number of oscillation increases gradually with $\Delta t$. For large $\Delta t$ the $\cos (\omega \Delta t)$ factor becomes a rapidly oscillating function, and the shape of the profile approaches that of the $\Delta t=0$ profile. In the present case, negative (positive) $\Delta t$ delays would be equivalent to a negative (positive) chirp in a single pulse laser field. It is noted, however, that since $\cos (-\omega \Delta t)=\cos (\omega \Delta t)$, one obtains the same $\left|\tilde{\mathcal{E}}_{\text {pump }}(\omega)\right|^{2}$ profile for equal time delays between the pulses, regardless their sign.

The spectral profiles of Fig. 3(b) show that phase modulation generated by simply varying $\Delta t$ produces substantial changes in the shape of the profile when the pulses overlap in the frequency domain. Such changes in the profile shape are expected to produce corresponding changes in the asymptotic final photofragment distributions. To illustrate this point, the laser field of eqn (1) has been applied with a temporal width of $\tau_{F W H M}=10 \mathrm{ps}$ for the two pulses in order to prepare the

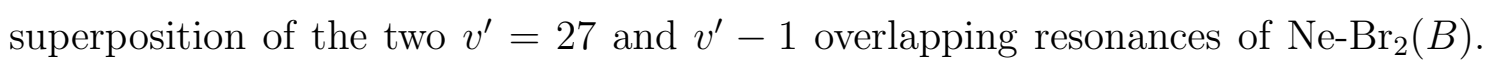
The predissociation dynamics was simulated and the $\operatorname{Br}_{2}\left(B, v_{f}\right)$ product fragment populations were calculated. This has been done for several time delays, specifically 
$\Delta t=0,10,20,25,30,35,40,50,75,100,150,200,250,300,400$, and 500 ps. In addition, several simulations have been carried out for negative time delays $(\Delta t=-10,-20,-30,-40,-50,-100,-200$, and -400$)$ in order to check that the same asymptotic populations are obtained as those found with the corresponding positive $\Delta t$ values. Indeed this was confirmed in all cases.

In Fig. 4(a) the $v_{f}=v^{\prime}-1$ normalized vibrational populations obtained for some of the $\Delta t$ values used are displayed. The figure shows that indeed the populations calculated with different $|\Delta t|$ values reach different asymptotic values in the longtime limit. The populations for $\Delta t=-30$ and $\Delta t=30$ are shown in the figure, and they coincide in the asymptotic limit, as discussed above. Interestingly, however, the transient populations for $\Delta t=-30$ and $\Delta t=30$ are remarkbly different before the asymptotic limt. This result is due to the fact that the two resonances of the superposition interfere themeselves, and the interference mechanism depends on the temporal order in which they are excited. ${ }^{32}$ The temporal order of resonance excitation for $\Delta t=-30$ (equivalent to a negative chirp) is different than for $\Delta t=30$ (equivalent to a positive chirp), producing a different interference pattern which is reflected in different transient population curves. It is noted that these phase modulation effects on the transient populations due to interference are appreciable until nearly $t=600 \mathrm{ps}$. Taking into account that for both $\Delta t=-30$ and $\Delta t=30$ the two pulses of the laser field are over before $t=50 \mathrm{ps}$ (see Fig. 3(a)), we find a time window of postpulse phase modulation effects due to interference of about 500 ps, much longer than that of 200 ps previously found with a single laser pulse. ${ }^{32,33}$

In Fig. 4(b) the asymptotic $P_{v_{f}=v^{\prime}-1}^{n o r m}(t \rightarrow \infty)$ populations are shown for the different time delays applied. The behavior of the values of the asymptotic population is symmetrical with respect to $\Delta t=0$, due to the even character of the $\cos (\omega \Delta t)$ function, as commented above. Sharp oscillations are found for low values of $\Delta t$, in the range $|\Delta t|<50$. In that range the asymptotic population changes from 0.105 to $\sim 0.135$, which involves a variation of nearly $30 \%$. The sharp oscillations leading to the largest variation of the population are a consequence of the large 
changes occurred in the $\left|\tilde{\mathcal{E}}_{\text {pump }}(\omega)\right|^{2}$ profile when $\Delta t$ is varied in the range of small time delays (see Fig. 3(b)). For $|\Delta t|>50$ the asymptotic population still displays oscillations, but they gradually decrease in intensity as $\Delta t$ increases, with the population converging to a value similar to that obtained for $\Delta t=0(\sim 0.12)$. Again, the reason of this convergence is that with increasing $\Delta t$ the highly oscillating shape of $\left|\tilde{\mathcal{E}}_{\text {pump }}(\omega)\right|^{2}$ approaches that of the profile obtained with $\Delta t=0$.

The results of Fig. 4(b) show that variations of nearly $30 \%$ can be produced in the asymptotic photofragment populations obtained from a superposition of only two overlapping resonances (the simplest superposition state), by simply varying the time delay between the two pulses of the laser field in a relatively small range of time. Thus the present phase modulation control scheme is both straightforward to carry out experimentally and powerful in order to achieve effective control. On the one hand, the experimental implementation of the control scheme only involves the use of two pump pulses with a time delay between them, which is easily achieved in current pump-probe experiments. On the other hand, the phase modulation produced by small time delays between the pulses cause large changes in the shape of the spectral profile and therefore in the population of the resonances in the superposition created. This fine selectivity of the population excited to all the superposition resonances leads to a large degree of control on the final product state distributions produced.

The effects of phase modulation of the $\left|\tilde{\mathcal{E}}_{\text {pump }}(\omega)\right|^{2}$ profile when the condition of frequency overlap between the two pulses of $\mathcal{E}_{\text {pump }}(t)$ is fulfilled should also be present in the asymptotic fragment state distributions obtained from a superposition of nonoverlapping, orthogonal states which cannot interfere themselves. In order to illustrate this point, the same laser field of Fig. 3 (i.e., using a temporal width of $\tau_{F W H M}=10 \mathrm{ps}$ for the two pulses of $\left.\mathcal{E}_{\text {pump }}(t)\right)$ is used to prepare a superposition of intermolecular essentially nonoverlapping resonances of $\mathrm{Ne}-\mathrm{Br}_{2}\left(B, v^{\prime}=16\right)$. The main goal here is to investigate how excitation of orthogonal, nonoverlapping states instead of overlapping ones in the superposition prepared affects the phase modulation control effects achieved on the asymptotic fragment state distributions, by 
comparison with the results of Fig. 4. Therefore, in order to perform a reliable comparison, the same laser field as that of Fig. 3, with the same separation between pulse central frequencies, $\omega_{1}-\omega_{2}=1.2 \mathrm{~cm}^{-1}$, has been used. More specifically, the $\omega_{1}$ frequency is tuned to excite the $n^{\prime}=8$ intermolecular resonance of $\mathrm{Ne}^{-\mathrm{Br}_{2}}\left(B, v^{\prime}=16\right)$, located at an energy of $24.95 \mathrm{~cm}^{-1}$ below the $\operatorname{Br}_{2}\left(B, v^{\prime}=16, j^{\prime}=0\right)+$ Ne dissociation threshold (then $\omega_{2}$ is tuned to an energy $1.2 \mathrm{~cm}^{-1}$ higher). Taking into account the spectral bandwith of $\left|\tilde{\mathcal{E}}_{\text {pump }}(\omega)\right|^{2}$ (see Fig. 3(b)), two resonances adjacent to $n^{\prime}=8$ are additionally populated in the superposition, namely $n^{\prime}=7$ and $n^{\prime}=9$, located at 26.96 and $22.39 \mathrm{~cm}^{-1}$, respectively. Thus, in this case a superposition of three nonoverlapping, rather narrow resonances is prepared.

Simulations of the predissociation dynamics of the $\mathrm{Ne}_{-} \mathrm{Br}_{2}\left(B, v^{\prime}=16\right)$ superpositon state have been carried out by applying the $\mathcal{E}_{\text {pump }}(t)$ field with different positive time delays, namely $\Delta t=0,10,20,25,30,35,40,50,100,150,200,300$, and 400 ps, and also several negative $\Delta t$ values. In Fig. 5(a) the transient population curves obtained for some of the time delays applied are presented. Indeed it is found that also for a superposition of orthogonal, nonoverlapping states the asymptotic fragment distributions can be controlled by means of the phase modulation induced by varying the time delay between the pulses, as expected. The comparison between the transient curves obtained for $\Delta t=-30$ and 30 ps displays an interesting result. The two curves converge to the same asymptotic value by around $t=300 \mathrm{ps}$, while the two corresponding curves in the case of the $v^{\prime}=27$ and $v^{\prime}-1$ overlapping resonances took about 600 ps to converge to their common asymptotic value (see Fig. 4(a)). Since the $\mathrm{Ne}_{-} \mathrm{Br}_{2}\left(B, v^{\prime}=16\right)$ nonoverlapping resonances are narrower (and therefore longer-lived) than the two $v^{\prime}=27$ and $v^{\prime}-1$ overlapping ones, the above difference in the size of the time window of postpulse effects cannot be due to the corresponding lifetimes of the resonances involved, which should contribute in the opposite direction. The time difference is actually due to the effect of interference between the overlapping resonances, absent in the case of the nonoverlapping resonances. A similar result was found previously when comparing the transient 
populations obtained from the $v^{\prime}=27$ and $v^{\prime}-1$ resonance superposition and from a $\mathrm{Ne}_{-} \mathrm{Br}_{2}\left(B, v^{\prime}=16\right)$ nonoverlapping resonance superposition. ${ }^{32}$

The value of the asymptotic normalized population $P_{v_{f}=v^{\prime}-1}^{n o r m}(t \rightarrow \infty)$ is plotted in Fig. 5(b) versus the different time delays applied. The behavior of the asymptotic population for $v^{\prime}=16$ is very similar to that found in Fig. 4(b), namely sharp oscillations for small time delays which gradually vanish as $\Delta t$ increases, reaching the asymptotic population for large $\Delta t$ a value close to that of $\Delta t=0$. The similarity of behaviors is not surprising, since it is determined by the shape of the $\left|\tilde{\mathcal{E}}_{\text {pump }}(\omega)\right|^{2}$ spectral profile, which is the same for equal $\Delta t$. The most interesting result is that the variation of the asymptotic population in Fig. 5(b) is much smaller than in Fig. 4(b), from $\sim 0.915$ to $\sim 0.923$, only nearly $1 \%$ compared to about $30 \%$ in the case of the overlapping resonances. The reason of this much smaller effect of the phase modulation control in the case of the narrow, nonoverlapping resonances is that in this case a substantially smaller amount of energy components are populated in the superposition prepared, and therefore this superposition is much less sensitive to changes in the $\left|\tilde{\mathcal{E}}_{\text {pump }}(\omega)\right|^{2}$ profile, as compared to the overlapping resonance superposition. Thus, overlapping resonances, or overlapping states in general, appear to be better candidates than orthogonal states to maximize the phase modulation control effects achieved on the fragment distributions with the present control scheme, both at intermediate postpulse times (due to interference between the states) and at asymptotic times.

\section{Summary and conclusions}

In conclusion, this work reports the first application to a polyatomic molecule of a coherent control scheme based on pure phase modulation of the pump laser field in the weak-field regime, in order to modify the asymptotic final state distributions of photodissociation fragments. The control scheme applies a pump laser field consisting of two pulses delayed in time. By varying the time delay between the pulses, phase modulation of the spectral bandwidth profile of the laser field can be achieved. 
The equations underlying the control scheme show that only when the bandwidths of the two pulses overlap in a range of the frequency domain, phase modulation can be effective in order to change the shape of the laser field spectral profile in that frequency range, leading to control effects on the asymptotic photofragment state distributions. The responsible for the phase modulation control effects is an interference term that appears in the expression of the spectral bandwidth profile when there is spectral overlap between the two pulses of the field. The magnitude of the range of spectral overlap between the pulses becomes an additional control parameter.

The performance of the control scheme is illustrated by preparing a superposition of two overlapping resonances with two different pump laser fields, consisting of two pulses nonoverlapping and overlapping in the frequency domain, respectively, and simulating its subsequent photodissociation dynamics. No phase modulation control effects are found at all on the asymptotic fragment populations in the case of spectrally nonoverlapping pulses, as predicted by the equations, while a variation of about $30 \%$ in the asymptotic distributions is obtained by varying the time delay between the pulses when they overlap in a frequency range. Furthermore, the same pump laser field with spectrally overlapping pulses is also applied to prepare a superposition of narrow, nonoverlapping resonances, and control effects on the asymptotic fragment populations are also achieved, although being much less intense. For simplicity, only two pulses have been included in the pump laser field applied here, but increasing the number of pulses might increase the flexibility and the degree of control achieved by the scheme.

Finally, the present findings are general for any superposition state of a polyatomic system, regardless the specific system of interest. The simplicity of the two conditions required for the laser field used in the present control scheme, namely two pulses that overlap in a certain range of frequencies and with a variable time delay between them, makes this scheme universal and straightforward to apply in current pump-probe experiments of molecular photodissociation processes. In this 
sense, following the spirit of previous experiments carried out on atomic and diatomic systems, ${ }^{10,35}$ a trivial experiment on a polyatomic molecule would consist of applying a pump laser field with two pulses with the same central frequency [i.e., $\omega_{1}=\omega_{2}$ in eqn (1)] and the same spectral width (then ensuring $100 \%$ of spectral overlap between them), and separated by a $\Delta t$ time delay. Thus a wide applicability of the control scheme proposed is envisioned.

\section{Acknowledgments}

This work was funded by the Ministerio de Ciencia e Innovación (Spain), Grant No. FIS2011-29596-C02-01, and COST Action program, Grant Nos. CM1401 and CM1405. The Centro de Supercomputación de Galicia (CESGA, Spain) is acknowledged for the use of its resources.

\section{References}

(1) S. A. Rice, M. Zhao, Optical Control of Molecular Dynamics, Wiley, New York, 2000.

(2) M. Shapiro and P. Brumer, Principles of the Quantum Control of Molecular Processes, Wiley, New York, 2003.

(3) D. J. Tannor, R. Kosloff and S. Rice, J. Chem. Phys., 1986, 85, 5805-5820.

(4) M. Shapiro, J. Phys. Chem. A, 1998, 102, 9570-9576.

(5) P. Anfinrud, R. de Vivie-Riedle and V. Engel, Proc. Natl. Acad. Sci. USA, 1999, 96, 8328-8329.

(6) A. Assion, T. Baumert, M. Bergt, T. Brixner, B. Kiefer, V. Seyfried, M. Strehle and G. Gerber, Science, 1998, 282, 919-922.

(7) T. C. Weinacht, J. Ahn and P. H. Bucksbaum, Nature, 1999, 397, 233-235.

(8) R. J. Levis, G. M. Menkir and H. Rabitz, Science, 2001, 292, 709-713.

(9) E. Skovsen, M. Machholm, T. Ejdrup, J. Thфgersen and H. Stapelfeldt, Phys. Rev. Lett., 2002, 89, 133004.

(10) M. Wellenhaupt, A. Assion, D. Liese, C. Sarpe-Tudoran, T. Baumert, S. 
Zamith, M. A. Boucheme, B. Girard, A. Flettner, U. Weichmann and G. Gerber, Phys. Rev. Lett., 2002, 89, 173001.

(11) C. Daniel, J. Full, L. González, C. Lupulescu, J. Manz, A. Merli, S. Vajda and L. Wöste, Science, 2003, 299, 536-539.

(12) M. Wollenhaupt, V. Engel, T. Baumert, Ann. Rev. Phys. Chem., 2005, 56, $25-56$.

(13) B. J. Sussman, D. Townsend, M. I. Ivanov and A. Stolow, Science, 2006, 314, 278-281.

(14) J. D. Farnum, G. Gidofalvi and D. A. Mazziotti, J. Chem. Phys., 2006, 124, 234103.

(15) H. Katsuki, H. Chiba, B. Girard, C. Meier and K. Ohmori, Science, 2006, 311, 15891592.

(16) G. Katz, M. A. Ratner and R. Kosloff, Phys. Rev. Lett., 2007, 98, 203006.

(17) E. A. Shapiro, I. A. Walmsley and M. Y. Ivanov, Phys. Rev. Lett., 2007, 98, 050501 .

(18) M. P. A. Branderhorst, P. Londero, P. Wasylczyk, C. Brif, R. L. Kosut, H. Rabitz and I. A. Walmsley, Science, 2008, 320, 638-643.

(19) G. Sansone et al., Nature, 2010, 465, 763.

(20) A. Biswas, M. Shapiro and P. Brumer, J. Chem. Phys., 2010, 133, 014103.

(21) O. Atabek, R. Lefebvre, M. Lepers, A. Jaouadi, O. Dulieu, V. Kokoouline, Phys. Rev. Lett., 2011, 106, 173002.

(22) Z. Hu, S. Singha, Y. Zhao, G. E. Barry, T. Seideman and R. J. Gordon, J. Phys. Chem. Lett., 2012, 3, 2744-2748.

(23) E. Wells, C. E. Rallis, M. Zohrabi, R. Siemering, B. Jochim, P. R. Andrews, U. Ablikim, B. Gaire, S. De, K. D. Carnes, B. Bergues, R. de Vivie-Riedle, M. F. Kling, and I. Ben-Itzhak, Nat. Commun., 2013, 4, 2895-2903.

(24) M. E. Corrales, J. González-Vázquez, G. Balerdi, I. R. Solá, R. de Nalda and L. Bañares, Nat. Chem., 2014, 6, 785-790.

(25) A. Palacios, A. González-Castrillo, and F. Martín, Proc. Natl. Acad. Sci. 
USA, 2014, 111, 3973-3978.

(26) P. Brumer and M. Shapiro, Chem. Phys., 1989, 139, 221.

(27) M. Shapiro and P. Brumer, J. Phys. Chem. A, 2001, 105, 2897.

(28) V. I. Prokhorenko, A. M. Nagy, S. A. Waschuk, L. S. Browun, R. R. Birge and R. J. D. Miller, Science, 2006, 313, 1257-1261.

(29) M. Spanner, C. A. Arango and P. Brumer, J. Chem. Phys., 2010, 133, 151101.

(30) C. C. Shu and N. E. Henriksen, J. Chem. Phys., 2011, 134, 164308.

(31) T. Grinev and P. Brumer, J. Chem. Phys., 2014, 140, 124307.

(32) A. García-Vela and N. E. Henriksen, J. Phys. Chem. Lett., 2015, 6, 824.

(33) A. García-Vela and N. E. Henriksen, Phys. Chem. Chem. Phys., 2016, 18, 4772 .

(34) A. K. Tiwari and N. E. Henriksen, J. Chem. Phys., 2016, 144, 014306.

(35) K. Ohmori, Y. Sato, E. E. Nikitin, and S. A. Rice, Phys. Rev. Lett., 2003, 91, 243003.

(36) M. Lein, M. Erdmann, and V. Engel, J. Chem. Phys., 2000, 113, 3609.

(37) J. A. Cabrera, C. R. Bieler, B. C. Olbricht, W. E. van der Veer and K. C. Janda, J. Chem. Phys., 2005, 123, 054311.

(38) M. A. Taylor, J. M. Pio, W. E. van der Veer and K. C. Janda, J. Chem. Phys., 2010, 132, 104309.

(39) T. A. Stephenson and N. Halberstadt, J. Chem. Phys., 2000, 112, 22652273 .

(40) A. García-Vela and K. C. Janda, J. Chem. Phys., 2006, 124, 034305.

(41) A. García-Vela, J. Chem. Phys., 2007, 126, 124306.

(42) A. García-Vela, J. Chem. Phys., 2008, 129, 094307.

(43) A. García-Vela, J. Chem. Phys., 2012, 136, 134304.

(44) A. García-Vela, Phys. Chem. Chem. Phys., 2015, 17, 29072-29078.

(45) A. García-Vela, J. Phys. Chem. Lett., 2012, 3, 1941-1945.

(46) A. García-Vela, J. Chem. Phys., 2013, 139, 134306. 
(47) A. García-Vela, RSC Adv., 2014, 4, 52174.

(48) F. Kelkensberg et al., Phys. Rev. Lett., 2009, 103, 123005.

(49) C. Lefebvre, T. T. Nguyen-Dang, F. Dion, M. J. J. Vrakking, V. N. Serov and O. Atabek, Phys. Rev. A, 2013, 88, 053416. 


\section{FIGURE CAPTIONS}

Fig. 1 (a) Gaussian temporal profiles of the envelopes of the two pump pulses of $\mathcal{E}_{\text {pump }}(t)$ with a temporal width $\tau_{F W H M}=100$ ps for different time delays $\Delta t=t_{2}-t_{1}$ between them. The other parameters of $\mathcal{E}_{\text {pump }}(t)$ are specified in the text. The center of one of the pulses is always fixed at $t_{1}=0$ ps. (b) Spectral bandwidth profile of the $\mathcal{E}_{\text {pump }}(t)$ field of panel (a) calculated using eqn (2). This profile is independent on the value of $\Delta t$.

Fig. 2 (a) Time-dependent normalized vibrational populations of the $\mathrm{Br}_{2}\left(B, v_{f}\right)$ fragment produced in the $v_{f}=v^{\prime}-1$ final vibrational state, upon predissociation of the $v^{\prime}=27$ and $v^{\prime}-1 \mathrm{Ne}-\mathrm{Br}_{2}\left(B, v^{\prime}\right)$ resonance superposition prepared when the $\mathcal{E}_{\text {pump }}(t)$ laser field with a pulse temporal width $\tau_{F W H M}=100$ ps is applied with different $\Delta t$ values. (b) Estimate of the effect of interference on the transient $v_{f}=v^{\prime}-1$ vibrational population, calculated as $\left(P_{v_{f}}^{n o r m}(t)-P_{v_{f}}^{n o r m, i n d}(t)\right) / P_{v_{f}}^{n o r m, i n d}(t)$ (see the text for details), for the transient population curves of panel (a).

Fig. 3 Gaussian temporal profiles of the envelopes of the two pump pulses of $\mathcal{E}_{\text {pump }}(t)$ with a temporal width $\tau_{F W H M}=10 \mathrm{ps}$ for different time delays $\Delta t$ between them. The other parameters of $\mathcal{E}_{\text {pump }}(t)$ are specified in the text. The center of one of the pulses is always fixed at $t_{1}=0$ ps. (b) Spectral bandwidth profiles of the $\mathcal{E}_{\text {pump }}(t)$ field of panel (a) calculated using eqn (2) for different time delays $\Delta t$.

Fig. 4 Time-dependent normalized vibrational populations of the $\operatorname{Br}_{2}\left(B, v_{f}\right)$ fragment produced in the $v_{f}=v^{\prime}-1$ final vibrational state, upon predissociation of the $v^{\prime}=27$ and $v^{\prime}-1 \mathrm{Ne}-\mathrm{Br}_{2}\left(B, v^{\prime}\right)$ resonance superposition prepared when the $\mathcal{E}_{\text {pump }}(t)$ laser field with a pulse temporal width $\tau_{F W H M}=10$ ps is applied with different $\Delta t$ values. (b) Asymptotic values of the normalized $\operatorname{Br}_{2}\left(B, v_{f}\right)$ fragment population produced in the $v_{f}=v^{\prime}-1$ final vibrational state, upon predissociation of the $v^{\prime}=27$ and $v^{\prime}-1 \mathrm{Ne}-\mathrm{Br}_{2}\left(B, v^{\prime}\right)$ resonance 
superposition prepared when the $\mathcal{E}_{\text {pump }}(t)$ laser field with a pulse temporal width $\tau_{F W H M}=10 \mathrm{ps}$ is applied with different $\Delta t$ values.

Fig. 5 Time-dependent normalized vibrational populations of the $\operatorname{Br}_{2}\left(B, v_{f}\right)$ fragment produced in the $v_{f}=v^{\prime}-1$ final vibrational state, upon predissociation of the $\mathrm{Ne}_{-} \mathrm{Br}_{2}\left(B, v^{\prime}=16\right)$ resonance superposition prepared when the $\mathcal{E}_{\text {pump }}(t)$ laser field with a pulse temporal width $\tau_{F W H M}=10 \mathrm{ps}$ is applied with different $\Delta t$ values. (b) Asymptotic values of the normalized $\operatorname{Br}_{2}\left(B, v_{f}\right)$ fragment population produced in the $v_{f}=v^{\prime}-1$ final vibrational state, upon predissociation of the $\mathrm{Ne}_{-} \mathrm{Br}_{2}\left(B, v^{\prime}=16\right)$ superposition prepared when the $\mathcal{E}_{\text {pump }}(t)$ laser field with a pulse temporal width $\tau_{F W H M}=10 \mathrm{ps}$ is applied with different $\Delta t$ values. 
Fig. 1, García-Vela
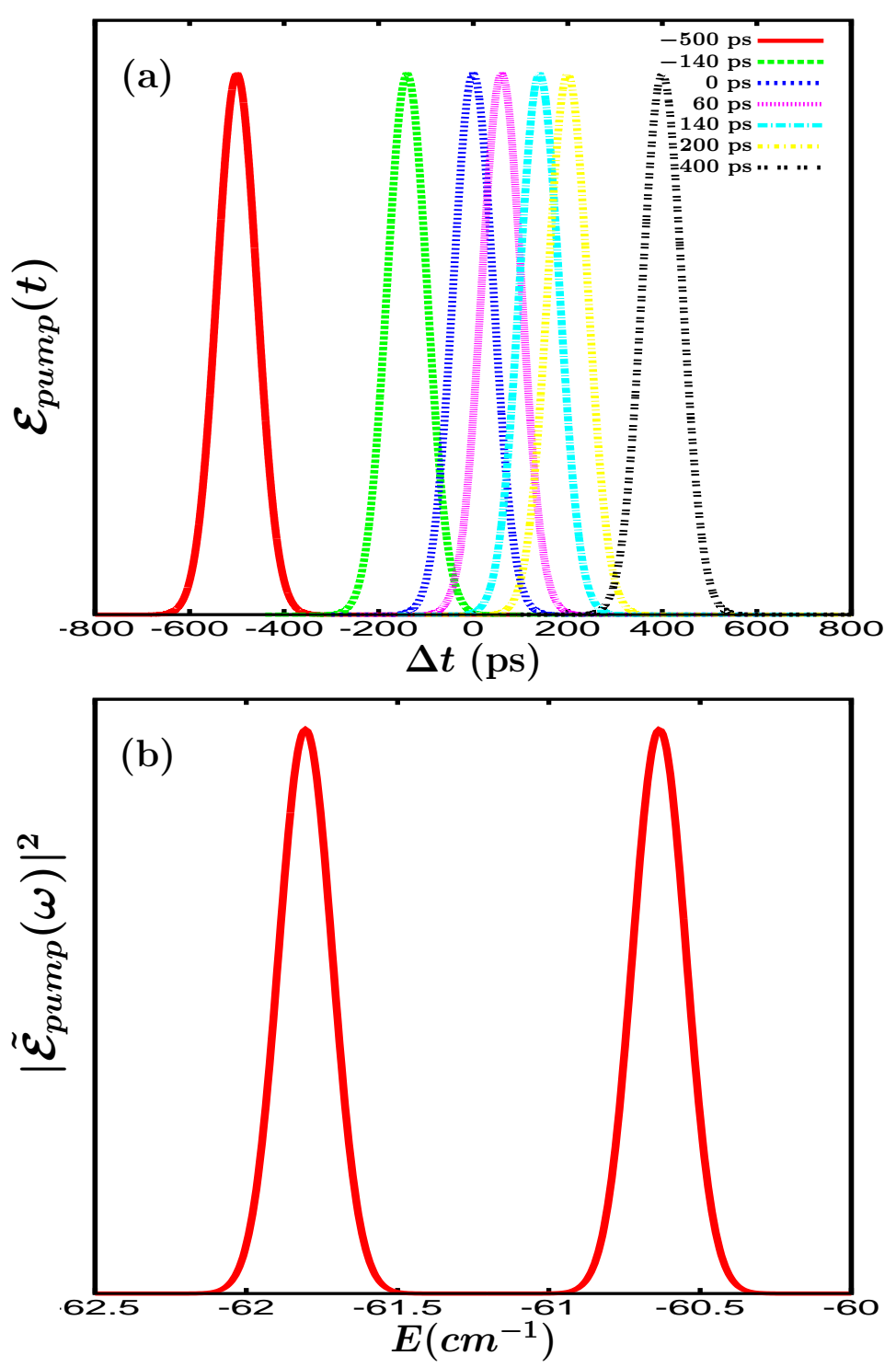
Fig. 2, García-Vela

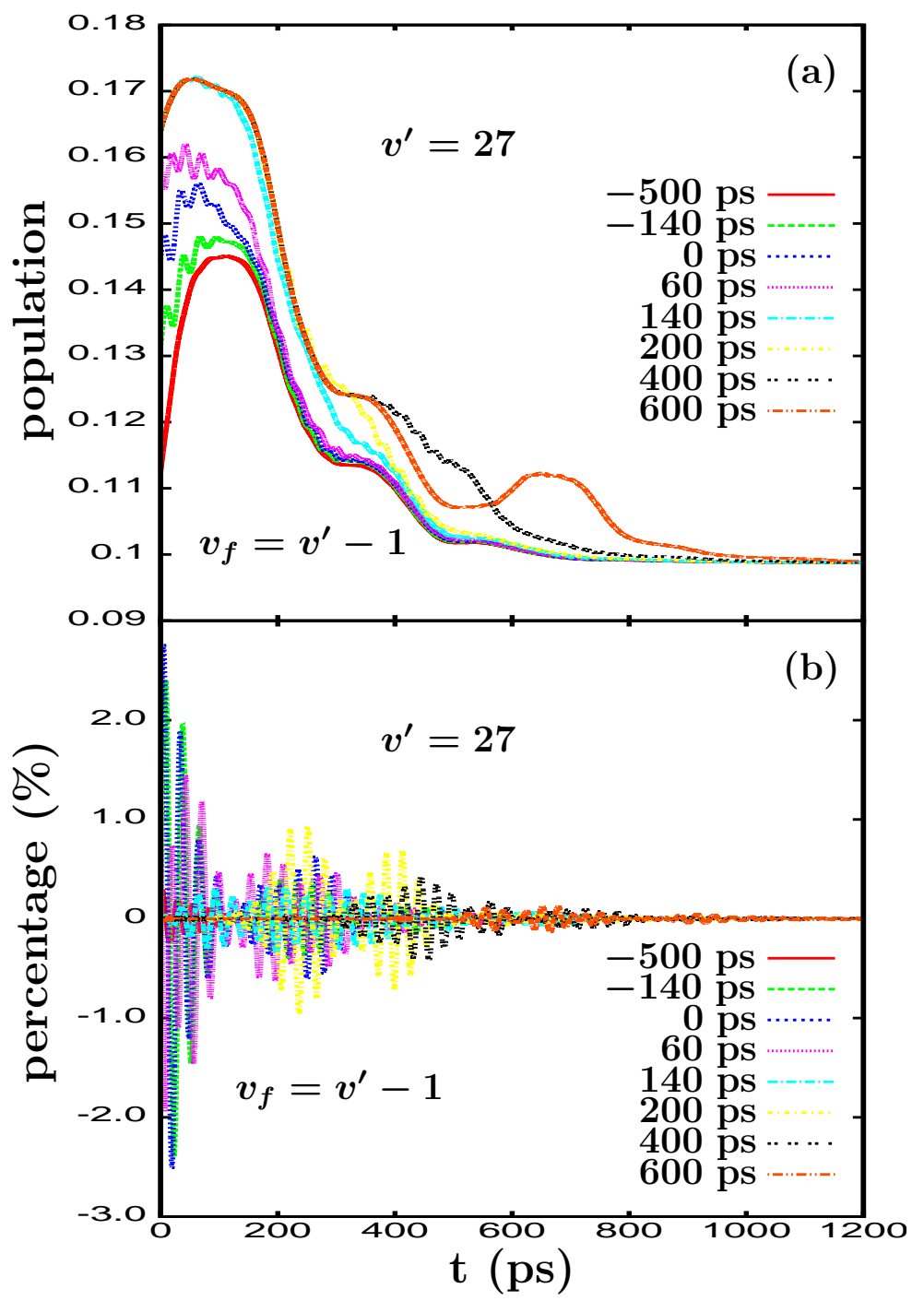


Fig. 3, García-Vela
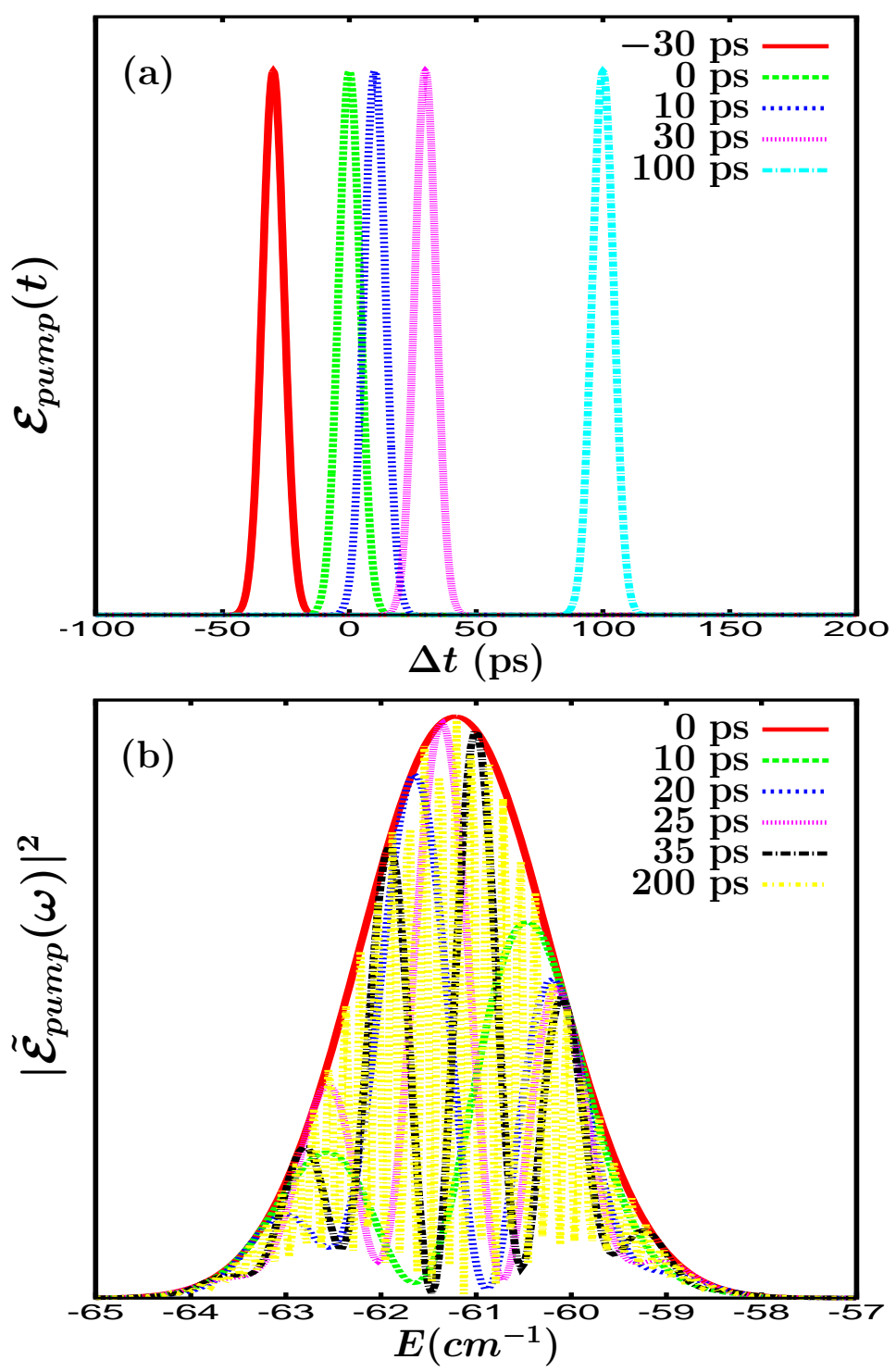
Fig. 4, García-Vela
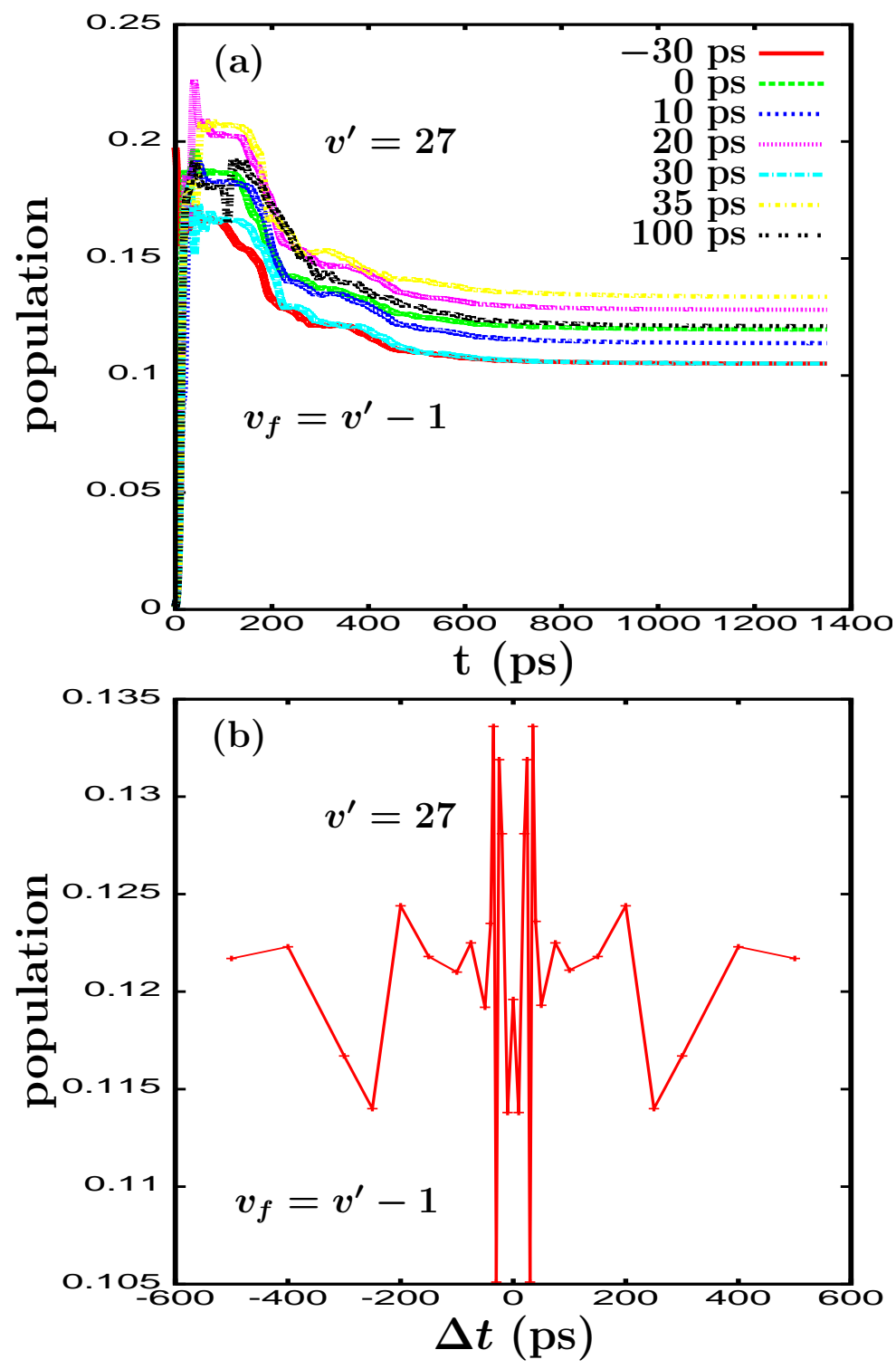
Fig. 5, García-Vela
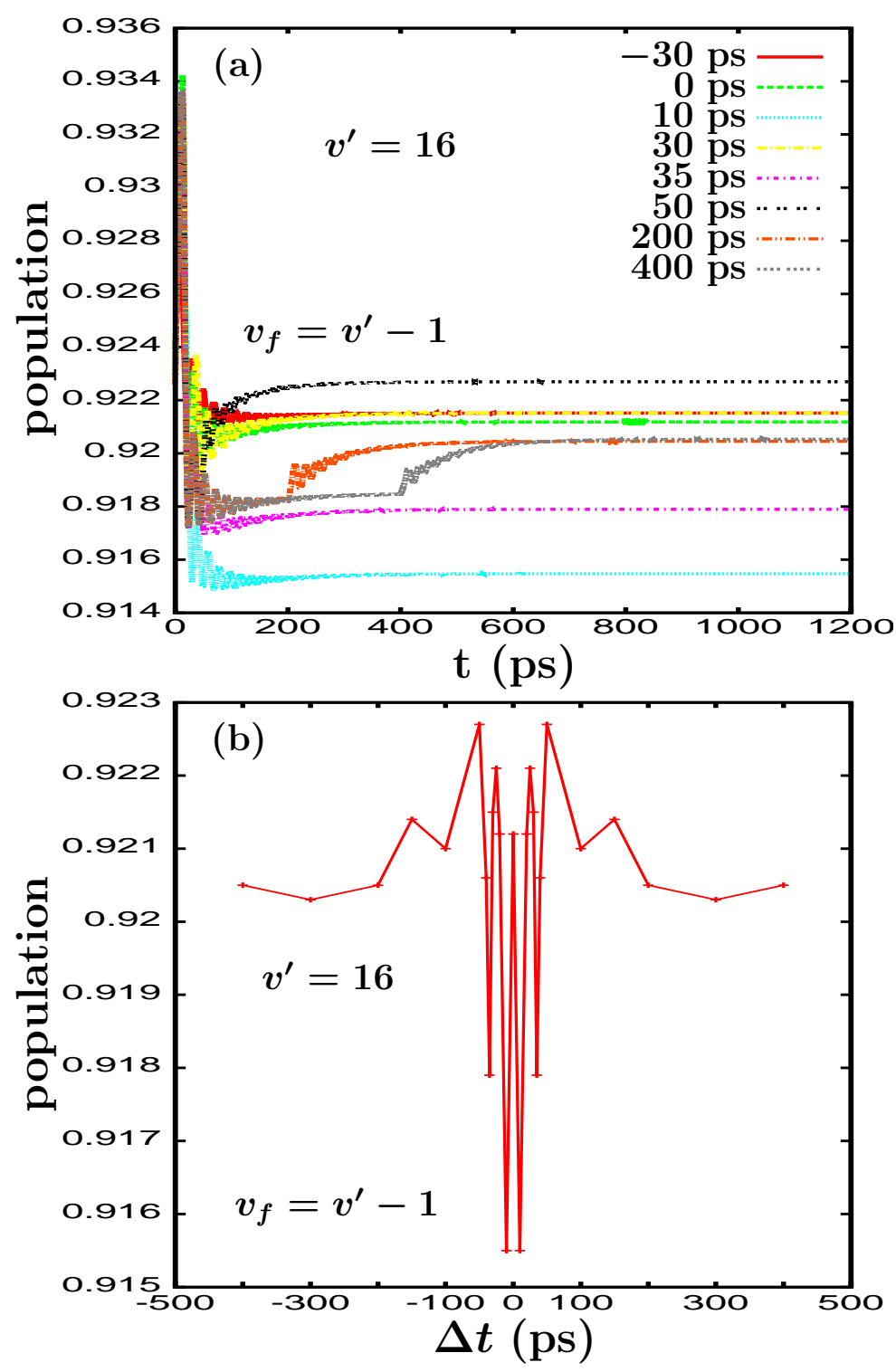